\title{
LA INFORMACIÓN EN LA ECONOMÍA DEL CONOCIMIENTO: RETOS Y OPORTUNIDADES PARA ESPAÑA
}

\section{Alberto Urueña-López y Antonio Hidalgo-Nuchera}

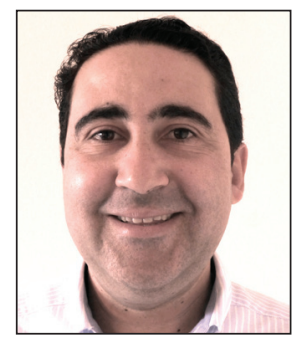

Alberto Urueña-López es subdirector adjunto del Observatorio Nacional de las Telecomunicaciones y de la Sociedad de la Información de Red.es. Doctor ingeniero de telecomunicación en el Programa de Economía y Gestión de la Innovación e ingeniero de telecomunicación por la Universidad Politécnica de Madrid (UPM), executive MBA por el Instituto de Empresa y máster en economía de las telecomunicaciones por la UNED. Investigador del Grupo Innopro de la UPM, sus líneas de trabajo son el estudio de la sociedad de la información y su impacto en ciudadanos y empresas. http://orcid.org/0000-0002-1299-2380

alberto.uruena@upm.es

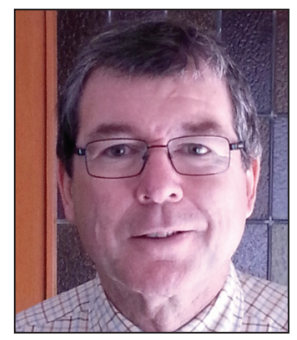

Antonio Hidalgo-Nuchera es catedrático de organización de empresas en la Escuela Técnica Superior de Ingenieros Industriales de la Universidad Politécnica de Madrid (UPM) y director del Grupo de Investigación en Innovación, Propiedad Industrial y Política Tecnológica (Innopro). Ha participado en proyectos financiados por la Comisión Europea y organismos nacionales sobre innovación tecnológica, transferencia de tecnología y desarrollo de políticas tecnológicas.

http://orcid.org/0000-0002-3598-9862

antonio.hidalgo@upm.es

Universidad Politécnica de Madrid ETSI Industriales, Depto. Ingeniería de Organización, Administración de Empresas y Estadísticas José Gutiérrez Abascal, 2. 28006 Madrid, España http://www.innopro.upm.es

\section{Resumen}

Partiendo del concepto de información, se revisa brevemente la definición del término economía del conocimiento. Se presentan dos líneas de evaluación: la primera, basada en la utilización de indicadores sintéticos (sobre un modelo de cuatro pilares: entorno económico y regulatorio, educación, infraestructuras TIC e innovación) y la segunda, de carácter más cuantitativo, utilizando técnicas económicas de contabilidad del conocimiento. Posteriormente se argumenta la utilización de un indicador sintético internacional existente que proporcione una perspectiva de los retos y oportunidades para la generación y utilización del conocimiento en España, complementado con los resultados de un reciente estudio sobre la difusión del conocimiento en distintos sectores productivos. Tras el análisis desde ambas perspectivas se obtienen unas conclusiones y recomendaciones que permitirían una utilización más óptima del conocimiento en España.

\section{Palabras clave}

Información, Economía del conocimiento, Indicadores sintéticos, Productividad, España.

Title: Information in the knowledge economy: challenges and opportunities for Spain

\begin{abstract}
Based on the concept of information, we begin with a brief review of the definition of the knowledge economy. There are two lines of evaluating the knowledge economy: the first is based on the use of summary indicators in a model with four pillars: regulatory environment, education, ICT infrastructure and innovation; the second is more quantitative, using economic techniques of accounting knowledge. We then defend the use of an existing international summary indicator that provides an overview of the challenges and opportunities for the generation and use of knowledge in Spain, enhanced by the results of a recent study on the dissemination of knowledge in various productive sectors. After completing the analysis from both perspectives, we obtain some conclusions and recommendations that would enable a more optimal use of knowledge in Spain.
\end{abstract}

\section{Keywords}

Information, Knowledge economy, Summary indicators, Productivity, Spain.

Urueña-López, Alberto; Hidalgo-Nuchera, Antonio (2013). “La información en la economía del conocimiento: retos y oportunidades para España". El profesional de la información, julio-agosto, v. 22, n. 4, pp. 339-345. 


\section{Introducción. Información y conocimiento}

Castells (1997) define la sociedad de la información como el estadio de desarrollo social caracterizado por la capacidad de sus miembros (ciudadanos, empresas y administración pública) para obtener y compartir cualquier información, instantáneamente, desde cualquier lugar y en la forma que se quiera, en grandes cantidades y a coste muy bajo.

Los conceptos de información y conocimiento presentan diferencias acusadas, aunque muchas veces se han utilizado con el mismo significado (Oxley et al., 2008). Sin embargo, cuando se extrae valor de la información o se utiliza de manera inteligente, se transforma en conocimiento a partir del cual se pueden tomar decisiones. El valor de una información y su transformación en un producto (bien o servicio) es lo que da su precio (Shapiro; Varian, 1999), convirtiéndola en el principal factor de producción, y desplazando a factores tradicionales en la economía clásica como el capital y la tierra (Drucker, 1994).

Existen múltiples definiciones del término economía del conocimiento (Oxley et al., 2008). La OCDE la define como aquella que se basa en la producción, distribución y uso del conocimiento y la información (OCDE, 1996). Su principal característica es que tiene una elevada dependencia de disponibilidad de información y de altos niveles de cualificación de sus trabajadores (OCDE; Eurostat, 2005). De manera similar, Kok (2004) señala que en las economías del conocimiento, la información y el saber acumulado tienen un papel preponderante en la creación de riqueza de su sistema productivo. Por tanto, resulta fundamental para la economía de un país cuantificar el valor económico de intangibles como la información y el conocimiento y analizar los factores que puedan permitir un mejor aprovechamiento de ambos (Ortiz-De-Urbina-Criado, 2003).

Cuando la información se utiliza de manera inteligente, se transforma en conocimiento a partir del cual se pueden tomar acciones y decisiones

\section{2. ¿Cómo medir la economía del conocimiento?}

Del análisis de la literatura científica relativa a la medición de la economía del conocimiento se identifican dos líneas específicas: La primera utiliza índices sintéticos y la segunda se fundamenta en instrumentos económicos basados en principios contables. De forma general, se puede afirmar que el enfoque con indicadores sintéticos proporciona una medida del potencial de generación y difusión del conocimiento en un país, mientras que el análisis a través de la contabilidad nos da una medida de la utilización real del conocimiento en las actividades productivas.

\subsection{Medición a través de indicadores sintéticos}

A través de un conjunto de indicadores simples ponderados, los índices sintéticos permiten obtener el potencial de utilización de la información y el conocimiento en un país.
Aunque este tipo de mediciones puede ser en algunos casos parcial, permiten obtener una valoración de los aspectos que favorecen o inhiben la utilización del conocimiento, lo que unido a que los esquemas basados en la contabilidad se encuentran en un estadio de desarrollo inicial, hacen de esta línea de medición la más utilizada en la actualidad.

Existen dos líneas de medición de la economía del conocimiento: utilizando índices sintéticos o a través de la contabilidad económica

Siendo conscientes del problema de simplificación que conlleva el diseño de cualquier indicador sintético (Paruolo et al., 2012), y dado el carácter global de la economía del conocimiento, es deseable que los indicadores sintéticos se elaboren con datos disponibles para un gran número de países y que permitan obtener diferencias con la finalidad de realizar análisis comparativos. A continuación se realiza un análisis de los principales indicadores sintéticos existentes que permiten realizar una aproximación del estado de la economía del conocimiento en España.

En 1999, el Programa de desarrollo económico del Banco Mundial (World Bank, 2012) elaboró una metodología que identifica las fortalezas y debilidades de un país respecto de sus competidores en la economía del conocimiento, conocido como el modelo de los cuatro pilares (gráfico 1) (Chen; Dahlman, 2005). Este modelo ha sido utilizado en el análisis de la economía española (Círculo de Empresarios, 2008). Tiene en cuenta la existencia de:

- infraestructura de comunicaciones que ayude a procesar y transmitir la información;

- trabajadores capacitados para crear, asimilar y difundir conocimiento de manera efectiva;

- sistema nacional de innovación formado por empresas, universidades y centros de investigación capaces de acceder al conocimiento global y aplicarlo generando valor.

- instituciones públicas capaces de incentivar y favorecer la difusión y utilización del conocimiento, fomentando y consolidando las interrelaciones entre el resto de los pilares.

La existencia de un desequilibrio en alguno de los cuatro pilares producirá un conjunto de disfunciones en la economía del conocimiento ya que éstos se encuentran relacionados entre sí.

Basándose en esta metodología, el Índice de la economía del conocimiento (conocido por sus siglas en inglés $K E I$, Knowledge economy index) mide la capacidad de un país para generar, adoptar y difundir el conocimiento teniendo en cuenta las facilidades ofrecidas por el entorno. Este índice se obtiene utilizando 12 indicadores simples (gráfico 2) y su principal inconveniente es que al utilizar variables muy sencillas (por ejemplo, en el caso de las TIC, número de teléfonos, ordenadores y usuarios de internet), el resultado no discrimina entre los países más competitivos y que utilizan 
la información y el conocimiento con mayor intensidad, por lo que no resulta muy útil para los países con mayor grado de desarrollo de su economía del conocimiento.

Desde 2004 el World Economic Forum elabora un informe anual de competitividad global en el que desarrolla el denominado Índice global de competitividad ( $\mathrm{GCl}$, en sus siglas en inglés) (World Economic Forum, 2012). Este indicador sintético está basado en doce categorías o pilares de la competitividad (gráfico 3) que ofrecen un panorama integral de las perspectivas de competitividad de un país:

- instituciones;

- infraestructura;

- entorno macroeconómico;

- educación primaria y salud;

- capacitación de la educación superior;

- eficacia de los mercados de bienes;

- eficacia laboral;

- desarrollo del mercado financiero;

- disponibilidad tecnológica;

- tamaño del mercado;

- sofisticación empresarial;

- innovación.

Cada uno de estos pilares está configurado por un número de indicadores básicos: las instituciones (públicas y privadas) tienen 22 indicadores; la educación superior, 8; las infraestructuras, 10, y la innovación 7 . Hay que resaltar que los indicadores de penetración TIC corresponden al pilar de infraestructuras dentro del esquema metodológico del Banco Mundial (líneas de telefonía fija y suscripciones de telefonía móvil).

La principal característica de este indicador es que tiene un carácter subjetivo, pues los datos se obtienen de encuestas a ejecutivos de empresas a los que se pide una valoración en una escala Likert de aspectos como la percepción de la situación económica, la valoración del gobierno y las instituciones públicas, la innovación y tecnología, el entorno financiero, la corrupción, la ética y la responsabilidad social, entre otros, lo que hace que los resultados dependan más de las opiniones de una muestra cualitativa no representativa estadísticamente que de datos cuantitativos.

Además de estos dos indicadores sintéticos existen otros que solamente proporcionan la medición de aspectos parciales de la economía del conocimiento, por lo que no pueden proporcionar un diagnóstico global.

El primer indicador es el denominado Networked readiness index (NRI) o Indicador de conectividad, ideado conjuntamente por el World Economic Forum y el Insead (Francia) y publicado en el informe The global information technology report (World Economic Forum; Insead, 2012), que recoge los perfiles sobre preparación tecnológica de más de 140 países. El NRI mide el uso que hacen las economías de las TIC y otras nuevas tecnologías para incrementar su crecimiento y su bienestar mediante el análisis del marco regulatorio que promueven las TIC, la preparación de los utilización de las mismas.

El segundo indicador es el denominado Global innovation index, elaborado por el Insead (Dutta, 2012), y orientado exclusivamente a la medición de la innovación (uno de los pilares de la economía del conocimiento).

El tercer indicador se denomina Índice de desarrollo TIC y ha sido desarrollado por la Unión Internacional de las Telecomunicaciones (UIT, 2011). Fue publicado en el informe sobre la medición de la sociedad de la información (Measuring the information society), que incluye el indicador sintético con datos para más de 150 países sobre su nivel de desarrollo TIC. Este indicador está formado a su vez por tres indicadores sintéticos o subíndices:

- subíndice de acceso TIC;

- subíndice de uso TIC;

- subíndice de habilidades TIC.

El enfoque con indicadores sintéticos nos da una medida del potencial de generación y difusión del conocimiento en un país

Su principal objetivo es evaluar y estudiar el desarrollo de la sociedad de la información en un país, así como monitorizar el progreso que se ha hecho para reducir la brecha digital. Al ser un indicador específico de TIC sólo es posible capturar aspectos relativos al acceso, uso y capacitación en estas tecnologías.

El diagnóstico con los datos disponibles a enero de 2013 utilizando el Índice global de competitividad del World Economic Forum revela que España ocupa el puesto 36 sobre un total de 144 países. Un análisis detallado del indicador pone de relieve la existencia de algunos problemas sobre los que es necesario llevar a cabo una reflexión: 
- Educación: los datos muestran deficiencias, pues con elevadas tasas de matriculación en educación secundaria y universitaria, la calidad del sistema educativo, en general, y de la educación en ciencias y matemáticas, en particular, nos sitúa en la tabla clasificatoria en los puestos 81 y 97, respectivamente, a pesar de la excelente opinión sobre las escuelas de negocio españolas que ocupan el cuarto puesto a nivel mundial. Se considera que la formación que se ofrece a los trabajadores por parte de las empresas es insuficiente, como lo demuestra la posición 105 que se ocupa.

- Régimen institucional: los resultados muestran que hay un exceso de regulación gubernamental (posición 120), que el gasto público no se realiza de manera eficiente (posición 108) y que el terrorismo supone costes adicionales para los negocios (posición 92). Dentro de este contexto el sector privado tampoco se libra de las críticas, ya que se considera que la eficacia de los consejos de administración es reducida (posición 97).

\section{El diagnóstico con indicadores sintéticos hace necesaria una reflexión: España ocupa el puesto 36 sobre un total de 144 países}

- Innovación: presenta deficiencias entre las que destaca que el gobierno no adopta productos de alta tecnología (posición 89), la escasa capacidad de innovación de nuestros agentes (posición 44), la débil colaboración entre la universidad y el sector empresarial (posición 45) y el pequeño volumen del gasto privado en I+D (posición 48).

- Infraestructuras de comunicaciones: su evaluación, por el contrario, presenta resultados más esperanzadores, y sólo destaca negativamente la penetración de la telefonía móvil (posición 55) y el uso de internet por los ciudadanos (posición 38). El resto de indicadores, como las suscripciones de banda ancha fija y móvil, y el ancho de banda por usuario, se encuentran mejor valorados ocupando las posiciones 14 y 27 , respectivamente.

\subsection{Medición a través de ins- trumentos contables}

Dentro de la línea de medición de la economía del conocimiento a través de instrumentos contables, diferentes estudios clasifican los sectores de la economía según los gastos en $\mathrm{I}+\mathrm{D}+\mathrm{i}$ realizados o el empleo de recursos humanos con alto grado de cualificación profesional (Pérez; Benages, 2012). Se- gún esta perspectiva, los países más avanzados en el uso de la información y del conocimiento son aquellos que cuentan con una estructura sectorial orientada a las actividades del conocimiento, lo que penaliza a países como España donde los sectores de I+D+i o de alta tecnología tienen un bajo peso en el PIB. Sin embargo, disponer de empresas o sectores intensivos en conocimiento solo indica un uso potencial del mismo, pero en ningún caso su efectiva utilización.

\section{Es necesario actuar en los sectores poco intensivos en conocimiento -como la construcción- fomentando su utilización}

Para solventar este problema, la medición de la intensidad del uso del conocimiento de una economía o, lo que es lo mismo, la cuantificación de la utilización efectiva del conocimiento en las actividades de producción económica está empezando a realizarse mediante lo que se denomina contabilidad del conocimiento o cuentas satélites del conocimiento. Consiste en medir el peso del conocimiento de un país en su PIB a través de la incorporación del conocimiento a las actividades de producción, estimando la utilización del conocimiento en maquinaria y equipo (incluyendo los equipos TIC) y del capital humano de manera separada. Una cuenta satélite a partir de los datos de la contabilidad nacional, permite obtener mediciones de la aportación de un sector o de determinadas actividades productivas a la economía a través de agregados como el PIB, la producción o el empleo. La complejidad y los requisitos de información necesarios para la creación de una cuenta satélite hacen que, hasta el momento, su utilización sea limitada en el caso español a los sectores de turismo y cultura, siendo la administración holandesa pionera en la cuantificación de la eco-

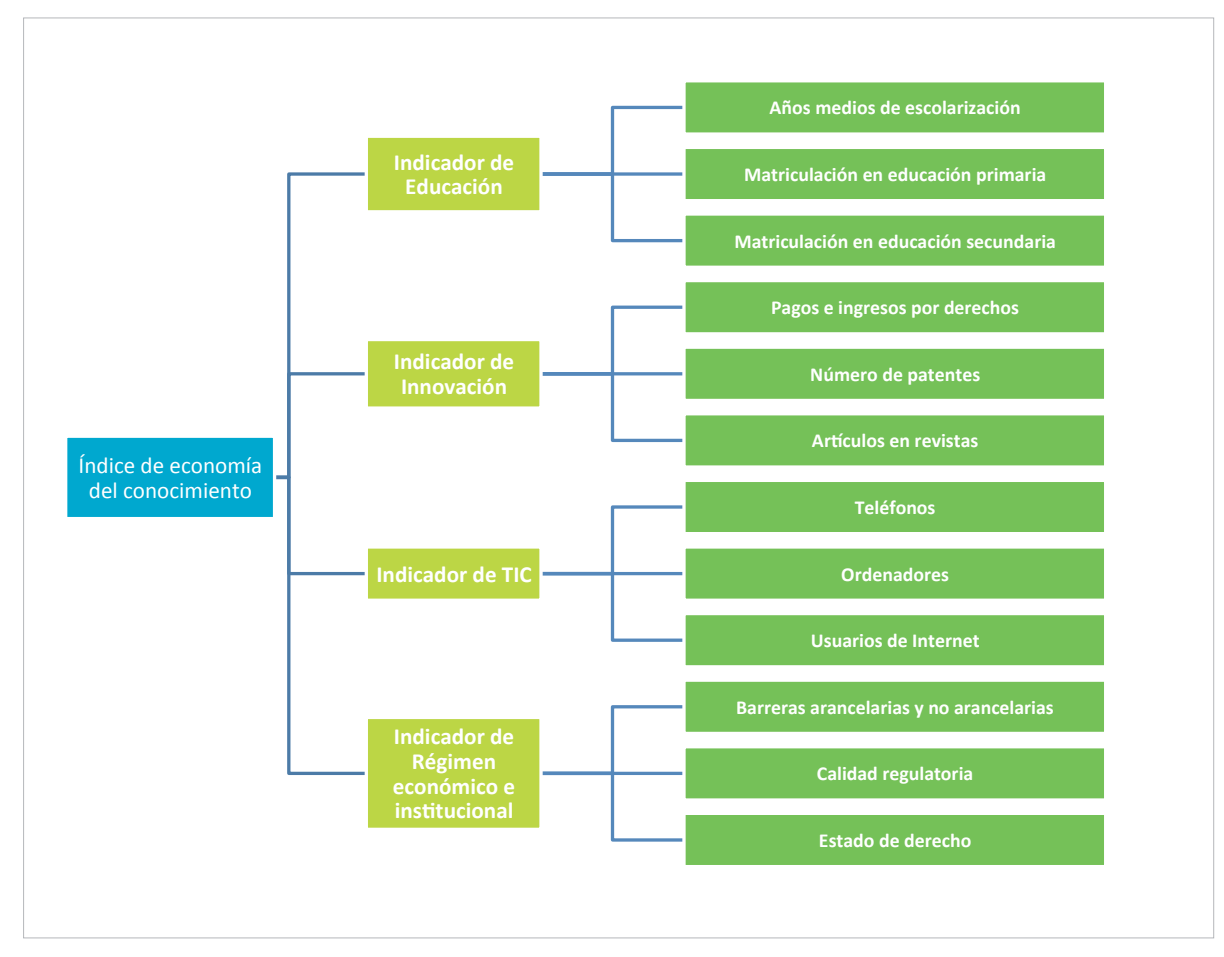

Gráfico 2. Indicadores del Knowledge economy index 
nomía del conocimiento a través de este instrumento.

Los resultados de uno de los primeros estudios que utiliza la contabilidad del conocimiento (Pérez; Benages, 2012) señalan que éste supone el $55 \%$ del PIB español, por lo que se puede decir que España es una economía basada en el conocimiento, aunque lejos de países líderes como Corea del Sur o Reino Unido en los que el conocimiento supera el $80 \%$ del PIB. No obstante, se ha realizado un importante esfuerzo en las últimas tres décadas, como pone de manifiesto el hecho de que el PIB basado en el conocimiento en España se ha multiplicado por un factor de 3,5 desde 1980. Las actividades que son más intensivas en el empleo de conocimiento, es decir, que utilizan capital humano con estudios superiores y TIC's son la educación, las actividades de fabricación de maquinaria, material eléctrico, electrónico y de equipos, y los sectores financiero, sanitario y administración pública (Pérez; Benages, 2012).

El estudio demuestra que el factor humano es el que más determina la diferencia de intensidad del uso de conocimiento inter e intra sectorialmente, y que además es el elemento más determinante en el aumento de la productividad (Pérez; Benages, 2012). Sin embargo, en muchas ocasiones se está dando un escaso aprovechamiento de las capacidades de los recursos humanos cualificados que realizan actividades que no dan valor a su conocimiento, lo que genera un bajo crecimiento de la productividad que, unido al aumento de la retribución de estos recursos, provoca una pérdida de competitividad. Por tanto, es más importante orientar hacia el conocimiento aquellos sectores poco intensivos en su utilización; es decir, y a modo de ejemplo, sería más interesante transformar el sector de la construcción que reducir drásticamente su peso en la economía.

El análisis a través de la contabilidad nos da una medida de la utilización real del conocimiento en las actividades productivas

\section{Conclusiones y recomendaciones para una utilización más efectiva del conocimiento en España}

El término recientemente acuñado de nueva economía o economía inmaterial, es decir, el desarrollo de toda la economía basada en la información y el conocimiento, y no en la producción de bienes industriales, está llamada a ser la gran especialización de los países desarrollados frente al resto del mundo en el siglo XXI. La economía del conocimiento, entendida como la utilización de información de valor en las actividades de los distintos sectores de la economía, puede llegar a representar un nuevo modelo económico sostenible a medio y largo plazo que ayude a superar la crisis actual. Este objetivo puede llegar a alcanzarse si se profundiza en los diferentes métodos que existen relativos a la medición de la economía del conocimiento.

La estructura de los cuatro pilares del Banco Mundial proporciona un esquema de análisis para medir el desarrollo de una economía del conocimiento

Desde el punto de vista de la contabilidad del conocimiento -como se ha dicho- se ha producido un notable aumento del peso del conocimiento en la economía española en los últimos 30 años. Sin embargo, y dado que el resto de países con los que España se debe comparar por el peso de su economía han continuado creciendo, es necesario llevar a cabo medidas que incrementen el peso del factor conocimiento en aquellos sectores poco intensivos en conocimiento, mediante el fomento de empresas innovadoras y otras medidas creativas en las que la administración pública tiene un papel fundamental.

Desde la perspectiva de los indicadores sintéticos, los resultados de los diagnósticos existentes no son muy positivos en el ámbito institucional, de educación y de innovación. Los principales problemas ligados al régimen institucional, como el exceso de regulación, la falta de eficacia en el gasto público y la negativa percepción de la acción institucional deben ser corregidos mediante acciones específicas. Respecto a este último problema, una medida interesante para la mejora de la percepción del sistema político y de gobierno sería utilizar internet y las nuevas tecnologías como medios para mostrar la transparencia del sistema. Otras 
medidas que pueden influir positivamente en la mejora de este indicador a nivel institucional podrían apoyar a los emprendedores en general, y al conocimiento en particular, reduciendo trámites burocráticos y aportando financiación e incentivos fiscales que atraigan el talento de jóvenes empresarios españoles. Hay que tener presente que de acuerdo con Drucker (2001), el conocimiento tiene características diferenciales: es acumulable, no tiene fronteras y es relativamente fácil fracasar en el emprendimiento ya que la información de partida con la que se cuenta es similar. Una regulación específica para los emprendedores que reconozca estos hechos diferenciales y aliente la creación de empresas de este tipo será un importante apoyo para posicionarse en la mente de los inversores como un país que reconoce la importancia del conocimiento.

Es necesaria una produnda reforma del régimen institucional, cambios en el sistema educativo y en el sistema de producción para la mejora en la utilización del conocimiento en España

La educación constituye un pilar básico para el desarrollo de una economía del conocimiento, pues se encuentra en la base del sistema. Sin embargo, los indicadores sintéticos muestran que en España se percibe una baja calidad en la misma a pesar de destacar por unas altas tasas de matriculaciones en secundaria y superior, y que las empresas invierten poco en la formación de sus empleados. Resulta necesario, por tanto, impulsar una reforma del sistema educativo orientada hacia la excelencia y una concienciación de las empresas sobre los resultados positivos de la inversión en formación y mejora de las capacidades de sus empleados. En este contexto parece necesario fomentar la generación de lo que puede denominarse como trabajadores del conocimiento y, especialmente, de "tecnólogos de la información y del conocimiento", es decir, trabajadores cuyos conocimientos teóricos se adquieran a través de una educación de calidad. La incentivación de esta educación dará lugar a la creación de nuevas industrias de futuro en nuestro país, intensivas en conocimiento, que proporcionarán empleabilidad al talento formado. La formación de especialistas en gestión de información, tratamiento de grandes volúmenes de datos, diseño de software, fabricación de productos utilizando impresoras 3D, etc., unido al uso transformador de las TIC en nuestras empresas (Ministerio de Industria, Turismo y Comercio, 2012) pueden suponer un verdadero impulso de la utilización efectiva del conocimiento en España.

Dentro de los indicadores relativos a la innovación, las estadísticas ponen de relieve que la universidad, que debería ser un elemento clave en el ecosistema del conocimiento, no actúa con eficacia en las tareas de difusión de la información y del conocimiento, formando un gran número de titulados no preparados con la calidad suficiente para la nueva economía del conocimiento. Además, la cooperación a través de los mecanismos de transferencia de tecnología entre los entornos científico y empresarial constituye una ne- cesidad explícita que necesita ser mejorada. En este sentido, y con la evidencia actual de que el proceso de innovación es un proceso en red, es decir, un proceso en el que participan todos los agentes económicos una eficaz aplicación de los mecanismos para transferir tecnología por parte del entorno científico constituye uno de los principales elementos estratégicos a la hora de consolidar la economía del conocimiento.

\section{Bibliografía}

Castells, Manuel (1997). "La era de la información, economía, sociedad y cultura. La sociedad red (volumen I)". Madrid: Alianza. ISBN: 9788420677002

Chen, Derek H. C.; Dahlman, Carl (2005). The knowledge economy, the KAM methodology and World Bank operations. World Bank Institute, working paper n. 37256.

http://siteresources.worldbank.org/KFDLP/Resources/ KAM_Paper_WP.pdf

Círculo de Empresarios (2008). Próxima estación: la economía del conocimiento

http://www.circulodeempresarios.org/es/publicaciones/ documentos-circulo/proxima-estacion-la-economia-delconocimiento

Drucker, Peter (1994). "The age of social transformation". The Athlantic monthly, Nov., v. 274, n. 5, pp. 53-80.

http://www.theatlantic.com/past/docs/issues/95dec/ chilearn/drucker.htm

Drucker, Peter (2001). "The next society". The economist, Nov.

http://www.economist.com/node/770819

Dutta, Soumitra (2012). "The global innovation index 2012: stronger innovation linkages for global growth". Fontainebleau (France): Insead. ISBN: 9782952221023

http://www.wipo.int/export/sites/www/freepublications/ en/economics/gii/gii_2012.pdf

Kok, Wim (2004). Facing the challenge: the Lisbon strategy for growth and employment. Luxembourg: Office for Official Publications of the European Communities. ISBN: 92894 70542

http://goo.gl/S192K

Ministerio de Industria, Turismo y Comercio (2012). Propuesta de agenda digital para España.

http://www.agendadigital.gob.es/images/doc/ PropuestadeAgendaDigitalparaEspana.pdf

OCDE (1996). The knowledge-based economy. Paris: OCDE. http://www.oecd.org/science/sci-tech/1913021.pdf

OCDE; Eurostat (2005). Oslo manual. The measurement of scientific and technological activities: guidelines for collecting and interpreting innovation. Paris: OCDE. http://www.oecd.org/science/inno/2367580.pdf

Ortiz-De-Urbina-Criado, Marta (2003). “Medición y auditoría del capital intelectual". El profesional de la información," julio-agosto, v. 12, n. 4, pp. 282-289.

http://www.elprofesionaldelainformacion.com/ contenidos/2003/julio/4.pdf 
Oxley, Les; Walker, Paul; Thorns, David; Wang, Hong (2008). "The knowledge economy/society: the latest example of 'Measurement without theory'?" The journal of philosophical economics, v. II, n. 1, pp. 20-54.

Paruolo, Paolo; Saisana, Michaela; Saltelli, Andrea (2013). "Ratings and rankings: voodoo or science?" Journal of the Royal Statistical Society: series A (Statistics in society), v. 176, n. 3, pp. 609-634.

http://dx.doi.org/10.1111/j.1467-985X.2012.01059.x

http://arxiv.org/pdf/1104.3009.pdf

Pérez, Francisco; Benages, Eva (2012). El PIB basado en el conocimiento: Importancia y contribución al crecimiento. Valencia: Ivie.

http://www.observatorioabaco.es/biblioteca/docs/115_ ABACO_CONOCIMIENTO_2012.pdf

Shapiro, Carl; Varian, Hal (1999). Information rules: a strategic guide to the network economy. Boston: Harvard Busi- ness School Press. ISBN: 9780875848631.

UIT (2011). Measuring the information society 2011. Geneva: International Telecommunications Union.

http://www.itu.int/net/pressoffice/backgrounders/general/ $p d f / 5 . p d f$

World Bank (2012). Knowledge for development (K4D). Washington: World Bank.

http://goo.gl/OOKiX

World Economic Forum (2012). The global competitiveness report 2012-2013. Geneva: World Economic Forum.

ht t p : //www3.weforum . org/docs/WEF_ GlobalCompetitivenessReport_2012-13.pdf

World Economic Forum; INSEAD (2012). The global information technology report 2012: living in a hyperconnected world. Geneva: World Economic Forum.

http://www3.weforum.org/docs/Global_IT_Report_2012.pdf

\section{Directorio EXIT \\ (EXpertos en Tratamiento de la Información) http://directorioexit.info}

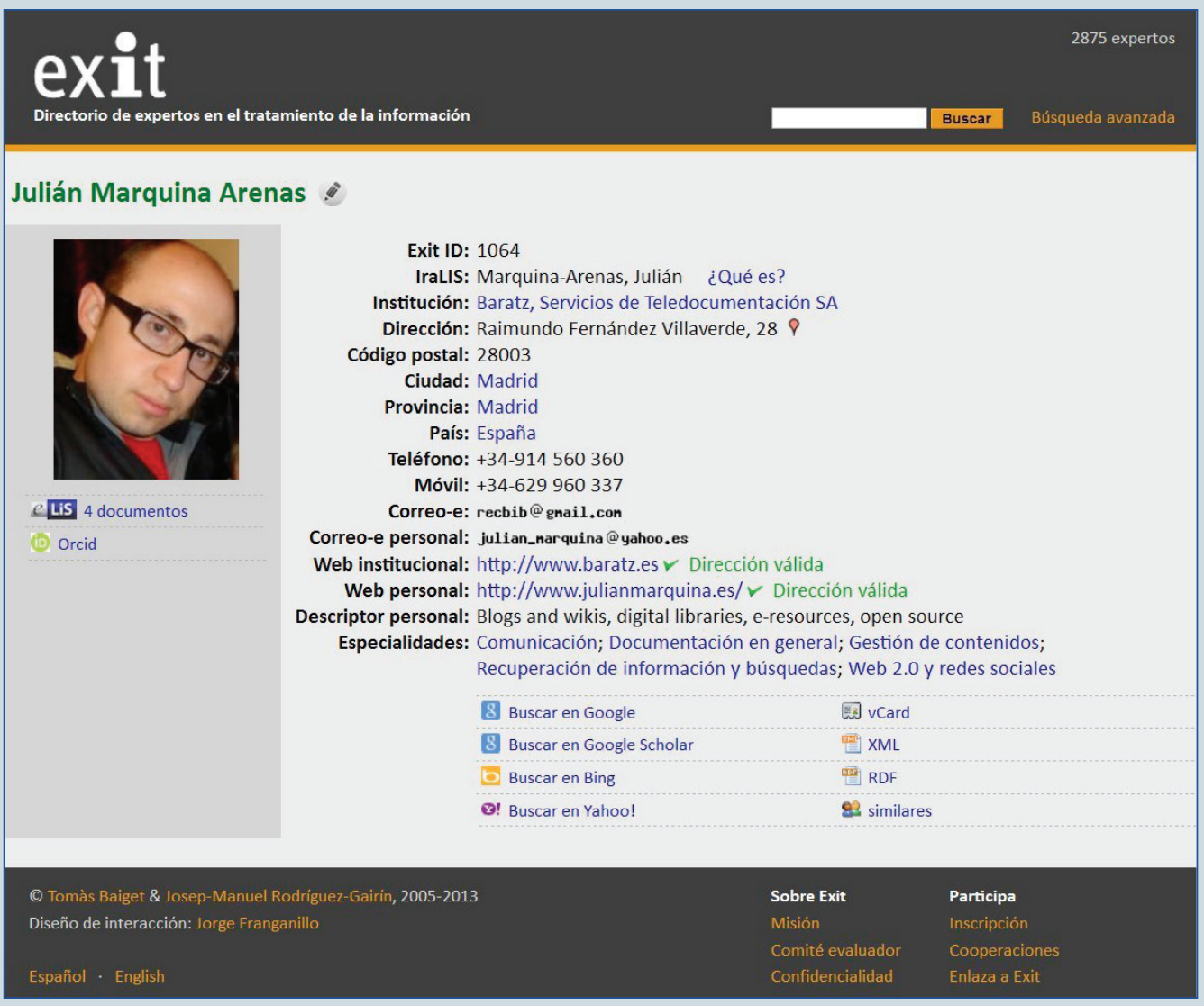

\section{Una ventana a las oportunidades profesionales}

La nueva versión 2013 localiza la dirección postal en Google maps y permite buscar perfiles de expertos similares a uno dado. 